\title{
On the existence of a mean on certain continua
}

by

\author{
Kermit Sigmon (Gainesville, Fla)
}

1. A topological groupoid is a Hausdorff space $G$ equipped with a continuous function $G \times G \rightarrow G$ whose value at $(x, y)$ will usually be denoted by $x y$. A topological groupoid $G$ is medial if $(x y)(u v)=(x u)(y v)$ for all $x, y, u, v \in G$, distributive if $x(y z)=(x y)(x z)$ for all $x, y, z \in G$, commutative if $x y=y x$ for all $x, y \in G$, and idempotent if $x x=x$ for all $x \in G$. A commutative, idempotent topological groupoid will be called a mean, as will its binary operation. The notions of mediality and distributivity are very natural ones and have proved useful in the study of mean values (see Frink [6] and Aczél [1], pp. 287 and 298). One easily shows that in the presence of idempotency, mediality implies distributivity.

Aumann [2], Eckmann [4] and Eckmann, Ganea and Hilton [5] have shown that there are wide classes of spaces which do not admit a mean. For instance, no $n$-sphere, $n \geqslant 1$, admits a mean. However, none of their methods apply, for example, to the space consisting of the curve $y=\sin \frac{1}{x}, 0<x \leqslant 1$, together with its limit continuum. It has been conjectured by A. D. Wallace that this space admits no mean but this remains open. In this paper we present certain results relevant to the admissibility of a mean on a continuum containing an open, dense halfline and give an example of a related space admitting no distributive mean. The action of a semigroup on such a space has been studied by Day and Wallace [3]. We conclude with the statement of some open problems.

It is a pleasure to acknowledge the direction by Professor A. D. Wallace of the research leading to this paper.

2. If $A$ and $B$ are subsets of a topological groupoid, we set

and let

$$
A B=\{a b \mid a \in A \text { and } b \in B\}
$$

and

$$
A^{(-1)} B=\{x \mid A x \cap B \neq \square\}
$$

$$
A^{[-1]} B=\{x \mid A x \subset B\}
$$


and, dually,

and

$$
B A^{(-1)}=\{x \mid x A \cap B \neq \square\}
$$

$$
B A^{[-1]}=\{x \mid x A \subset B\} .
$$

It should be noted that if $A$ is a singleton set, then $A^{(-1)} B=A^{[-1]} B$.

(2.1) LEMna. If $A$ and $B$ are subsets of a topolgical groupoid, then

(i) $A^{[-1]} B$ is open if $A$ is compact and $B$ is open, and dually.

(ii) $A^{(-1)} B$ is open if $B$ is open, and dually.

(iii) $\{x \mid A \subset A x\}$ is closed if $A$ is compact, and dually.

Proof. (i) If $x \in A^{[-1]} B$, then $A x \subset B$. But since $A$ is compact, it. follows from the continuity of the groupoid multiplication that there is an open set $V$ about $x$ such that $A V C B$. Hence $x \in V C A^{[-1]} B$ so that $A^{[-1]} B$ is open.

(ii) We have that $A^{(-1)} B=\bigcup\left\{a^{(-1)} B \mid a \in A\right\}=\bigcup\left\{a^{[-1]} B \mid a \in A\right\}$ and each of $a^{[-1]} B$ is open by (i).

(iii) Since $\{x \mid A \subset A x\}=\bigcap\left\{A^{(-1)} a \mid a \in A\right\}$ it suffices to show that $A^{(-1)} a$ is closed for each $a \in A$. If $x \notin A^{(-1)} a$, then $a \notin A x$ and hence $A x \subset X \backslash\{a\}$ which is open. Then, since $A$ is compact, there is an open set $V$ about $x$ such that $A V \subset X \backslash\{a\}$ so that $x \in V \subset X \backslash A^{(-1)} a$. It follows that $A^{(-1)} a$ is closed.

For the remainder of this section we suppose that $M$ is a continuum containing an open, dense half-line $W$ whose complement $C$ is non-degenerate and we let $\mathrm{P}$ be a property of topological spaces which is a continuous invariant on continua and which $M$ does not possess. Two examples of such a property are local connectivity and arewise connectivity.

(2.2) LemMa. (i) $C$ is a $C$-set (i.e., any continuum which intersects $C$ but is not contained in $C$, must contain $C$ ) and hence is connected.

(ii) Any subcontinuum of $M$ which has property $\mathrm{P}$ and which inter. sects $C$ must be contained in $C$.

Proof. (i) It is easy to see that $C$ is a $C$-set and the fact that $C$ is connected follows from lemma 1 of [8].

(ii) If, on the contrary, $K$ is a subcontinuum of $M$ having property $\mathrm{P}$ and intersecting both $C$ and $W$, then by part (i) $C \subset K$ and hence, since $K \cap W \neq \square, K \stackrel{t}{=} M$. But since $\mathrm{P}$ is a continuous invariant on continua, this implies that $M$ has property $\mathrm{P}$ contrary to our assumption.

The next theorem shows that the multiplication of an idempotent topological groupoid on $M$ must "spread" near $C$. A subset $J$ of a topological groupoid $G$ is a right ideal of $G$ if $J G C J$, a left ideal of $G$ if $G J \subset J$, and an ideal of $G$ if $J G \cup G J \subset J$.
(2.3) THEOREM. If $M$ is an idempotent topological groupoid, then W is a subgroupoid of $M$ and either $C \subset O x$ for all $x \in C$ or $C \subset x C$ for all $x \in C$. The first case holds whenever either $C$ or $W$ is a right ideal of $M$, and the second case holds whenever either $C$ or $W$ is a left ideal of $M$. If, in addition, $C$ has property $\mathrm{P}$, then $C$ is a subgroupoid of $M$, either $C$ is a right ideal or $W$ is a left ideal of $M$ and, dually, either $C$ is a left ideal or $W$ is a right ideal of $M$.

Proof. The theorem is contained in the following series of assertions. The proofs of the primed assertions, which are dual to the unprimed ones, are omitted.

(i) $W$ is a subgroupoid of $M$. We know that $W^{2}$ is arcwise connected since $W$ is and that $W C W^{2}$ from the idempotency so that observing that $W$ is an arc-component of $M$ it follows that $W^{2}=W$.

(ii) $M=W^{[-1]} W \cup W^{[-1]} C$. Since $W$ is an arc-component of $M$ and $W x$ is arcwise connected for each $x \in M$, we must have that, for each $x \in M$, either $W x \subset W$ or $W x \subset C$. This shows that $M \subset W^{[-1]} W \cup W^{[-1]} C$ and the reverse containment is trivial.

(ii) $M=W W^{[-1]} \cup^{\prime} C W^{[-1]}$.

(iii) If $C \subset W^{[-1]} W$, then $C \subset C x$ for all $x \in C$ and if $C \not \subset W^{[-1]} W$, then $C C x C$ for all $x \in M$. First, suppose that $C \subset W^{[-1]} W$ and let $x \in C$. Then $M x$ is a continuum intersecting $C$, since $x \in M x$, but not contained in $C$, because $x \in C \subset: W^{[-1]} W$ and hence $W x \subset W \cap M x$. It follows from lemma (2.2(i)) that $C \subset M x$ and since $W x \subset W$, we must have that $C \subset C x$.

Next, suppose that $C \not \subset W^{[-1]} W$ so that, in view of (ii), we have that $C \cap W^{[-1]} C \neq \square$. If we choose $x_{0} \in C \cap W^{[-1]} C$, then for $y \in W, y M$ is a continuum which intersects $C$, because $y x_{0} \in y C \cap W x_{0} \subset y M \cap C$, but which is not contained in $C$, since $y \in y M$, so that from lemma (2.2(i)) we have that $C \subset y M$ and hence $C \subset y C$, since $y W \subset W$. We have shown that $W C\{x \mid C \subset x C\}$. But, by lemma (2.1(iii)), $\{x \mid C \subset x C\}$ is closed from which it follows that $M=W^{*} C\{x \mid C C x C\}$.

(iii) $^{\prime}$ If $C \subset W W^{[-1]}$, then $C \subset x C$ for all $x \in C$ and if $C \not \subset W W^{[-1]}$, then $C \subset C x$ for all $x \in M$.

(iv) $C \subset W^{[-1]} W$ iff $W$ is a right deal of $M$ and $C \subset W^{[-1]} C$ iff $C$ is a left ideal of $M$.

It is clear that $C \subset W^{[-1]} W$ iff $W C \subset W$, but since $W^{2} \subset W$, we have that $W C \subset W$ is equivalent to $W M \subset W$. Similarly, $C \subset W^{[-1]} C$ iff $W C \subset C$ which, in turn, is equivalent to $M C \subset C$, since $C$ is closed and $W^{*}=M$.

(iv) $C \subset W W^{[-1]}$ iff $W$ is a left ideal of $M$ and $C \subset C W^{[-1]}$ iff $C$ is a right ideal of $M$. 
(v) $O C O x$ for all $x \in C$, whenever either $C$ or $W$ is a right ideal of $M$ and $C \subset x C$ for all $x \in C$, whenever either $C$ or $W$ is a left ideal of $M$. Observing that $C \subset W^{[-1]} C$ implies $C \not \subset W^{[-1]} W$ (and that $C \subset C W^{[-1]}$ implies $O \not \subset W W^{[-1]}$ ) we see that (v) follows from combining (iii), (iii)', (iv) and (iv)'.

(vi) If $C$ has property $\mathrm{P}$, then $C$ is a subgroupoid of $M$. If $x \in C$, then $x \in x C \cap C$ and $x C$ has property $\mathrm{P}$ so, by lemma (2.2(ii)), $x C \subset C$, i.e., $O^{2} \subset C$.

(vii) If $C$ has property $\mathrm{P}$, then either $C \subset W^{[-1]} W$ or $C \subset W^{[-1]} C$. If $C \not \subset W^{[-1]} W$ with, say, $x_{0} \in C \backslash W^{[-1]} W$, then in view of $2.2(\mathrm{i})$ we have that $W x_{0} \subset C$ so that, for each $y \in W, y x_{0} \in y O \cap O$ and hence from the fact that $y C$ has property $\mathrm{P}$ and from $(2.2$ (ii)) it follows that $y C \subset C$. This shows that $W C \subset C$ and hence $C \subset W^{[-1]} C$.

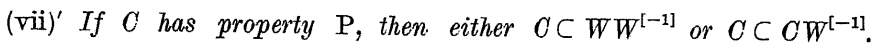
An immediate consequence of the preceding theorem is the following:

(2.4) Corollary. If $M$ is a mean, then $C \subset C x$ for all $x \in C, W$ is a submean of $M$ if, in addition, $C$ has property $\mathrm{P}$, then $C=C x$ for all $x \in C$
and either $C$ or $W$ is an ideal of $M$.

In the case of an idempotent semigroup, theorem (2.3) reduces to the following corollary. A semigroup is an associative topological groupoid and a topological semilattice is an associative mean.

(2.5) Corollary. If $M$ is an idempotent semigroup, then $C$ is a subsemigroup of $M$ such that either $x y=x$ for all $x, y \in C$ or $x y=y$ for all $x, y \in C$. Hence, $M$ does not admit the structure of a topological semilattice.

Proof. If $M$ is an idempotent semigroup, then, from theorem (2.3), we have that either $C \subset C x$ for all $x \in C$ or $C \subset x C$ for all $x \in C$ and hence, since each element of $O$ is idempotent, $O$ consists of either all right units or all left units of $C$. For, supposing $C \subset C x$ for all $x \in C$, if $x, y \in O$, then $x \in C \subset C y$ and hence $x=z y$ for some $z \in C$ from which it follows that $x y=(z y) y=z y^{2}=z y=x$. The dual assertion follows similarly.

If $\boldsymbol{M}$ were a topological semilattice, then from the above, each element of $C$ would be a unit for $C$, which would imply that $O$ is degenerate, con-
trary to our assumption.

3. Let $S$ be the subcontinuum of the plane consisting of the union
the sets of the sets

$$
C=\{(r, s) \mid r=0 \text { and }-1 \leqslant s \leqslant 1\}
$$

$$
W=\left\{(r, s) \mid s=\sin \frac{1}{r} \text { and } 0<r \leqslant 1\right\}
$$

and, for each positive integer $k$, let $A_{k}$ be the subcontinuum of $S$ given by

$$
A_{k}=\left\{(r, s) \in \mathbb{S} \mid \frac{2}{(4 k+3) \pi} \leqslant r \leqslant \frac{2}{(4 k+1) \pi}\right\} .
$$

It will be convenient to denote the subcontinuum of $S$ irreducible between subcontinua $A$ and $B$ of $S$ by $[A, B]$ and to let $\langle A, B]=[A, B] \backslash A$, etc.

We now foil your idea of an example of a mean on $S$.

(3.1) Proposition. S admits no mean which is internal on $W$, in the sense that $x y \in[x, y]$ whenever $x, y \in W$.

Proof. Suppose that, on the contrary, $S$ admits a mean such that $x y \in[x, y]$, whenever $x, y \in W$. Then the mean is internal on $C$. For suppose that $x_{1} x_{2} \notin\left[x_{1}, x_{2}\right]$ for some $x_{1}, x_{2} \in C$ and let $V$ be a spherical neighborhood (in the usual metric of the plane) of $x_{1} x_{2}$ such that $\nabla^{*} \cap\left\{x_{1}, x_{2}\right\}=\square$ and, using the continuity of the mean, let $U_{1}, U_{2}$ be spherical neighborhoods of $x_{1}, x_{2}$, respectively such that $U_{1} U_{2} \subset V$ and $\left(U_{1} \cup U_{2}\right) \cap$ $\cap V=\square$. We remind you that $U_{1} Z_{2}=\left\{x y \mid x \in D_{1}, y \in U_{2}\right\}$. If we choose $k$ so large that $A_{k} \cap U_{1} \neq \square \neq A_{k} \cap U_{2}$ and let $y_{1} \in A_{k} \cap U_{1}$ and $y_{2} \in A_{k} \cap$ $\cap U_{2}$, then by our choice of neighborhoods, $\left[y_{1}, y_{2}\right] \cap V=\square$. But $y_{1} y_{2} \in U_{1} U_{2} \subset V$ while from the internality of the mean on $W, y_{1} y_{2} \in\left[y_{1}, y_{2}\right]$, a contradiction. Hence, the mean is internal on $C$. Letting $a$ and $b$ denote the endpoints of 0 , we have from (2.4) that $a \epsilon C=C b$ so $a=x b$ for some $x \in O$. But from the above this is only possible if $x=a$, i.e., $a=a b$. Similarly, one shows that $b=a b$ and hence $a=b$, a contradiction. The proposition follows.

Next we show that any distributive (and hence any medial) mean on the sinusoidal continuum described above must have the limit continuum as an ideal and give an example of a continuum admitting no distributive mean. We first prove a lemma.

(3.2) LeMmia. If $A$ is a submean of a distributive mean $M$ then, for each $x \in M, A x$ is also a submean of $M$.

Proof. We have only to observe that if $A$ is a submean of $M$, then the distributivity of $M$ implies that

for all $x \in \mathbb{M}$.

$$
(A x)(A x)=A A \cdot x=A x
$$

A nodal set in a continuum is a proper, non-void subcontinuum having a one point boundary.

(3.3) Propostrton. If $S$ is a distributive mean then $C$ is an ideal of $S$.

Proof. In view of corollary (2.4) (with $M=S$ and $\mathrm{P}=$ local connectivity) we have only to show that $W$ is not an ideal of $S$. To this end we suppose, on the contrary, that $W$ is an ideal of $S$ and note that, for 
each $y \in W, S y=\left[r_{y}, t_{y}\right]$ for some $r_{y}, t_{y} \in W$ and $C y=\left[e_{y}, f_{y}\right]$ for some $e_{y}, f_{y} \in W$, and we set $N_{0}=\left[C, r_{q}\right]$ where $q$ is the endpoint of $S$.

We now prove several assertions whose object is to show that the mean is internal on $C$.

(i) $N_{0} \cap W C\left\{r_{y} \mid y \in W\right\} \cap\left\{f_{y} \mid y \in W\right\}$. If we define $g: W \rightarrow W$ by $g(y)=r_{y}$, then $g$ is continuous, for if we let $\langle u, v\rangle$ be an open interval contained in $W$, let $U_{1}=[C, v\rangle$, and let $U_{2}=\langle u, q]$, then $U_{1}$ and $U_{2}$ are both open and, since $S$ is compact, so are $S^{(-1)} U_{1}$ and $S^{[-1]} U_{2}$. But $W$ is open and

$$
g^{-1}(\langle u, v\rangle)=S^{(-1)} U_{1} \frown S^{[-1]} U_{2} \frown W
$$

from which follows the continuity of $g$. If one defines $h: W \rightarrow W$ by $h(y)=f_{y}$ then one shows $h$ continuous in a similar manner. It follows that each of $g(W)$ and $h(W)$ is connected.

Now let $y \in N_{0} \cap W$ and observe that $S^{(-1)}[C, y\rangle$ and $C^{[-1]}[C, y\rangle$ are open, the latter because $C$ is compact, and that $C C C^{[-1]}[C, y\rangle \cap$ $\cap S^{(-1)}[C, y\rangle$ since, for $x \in C$, we have that $C x=C \subset[C, y\rangle$ and $x \in S x \cap$ $\cap C \subset S x \cap[C, y\rangle$. We may then choose an element $x \in C^{[-1]}[C, y\rangle \cap$ $\cap \mathcal{S}^{(-1)}[C, y\rangle \cap W$ so that, on the one hand, $C x C[C, y\rangle$ and hence $y \in\left[f_{x}, r_{q}\right] \subset\left[f_{x}, f_{q}\right] \subset h(W)=\left\{f_{y} \mid y \in W\right\}(h(W)$ is connected!) and, on the other hand, $S x \cap[C, y\rangle \neq \square$ so that $y \in\left[r_{x}, r_{q}\right] \subset g(W)=\left\{r_{y} \mid y \in W\right\}$.

(ii) If $h: W \rightarrow W$ is defined by $h(y)=f_{y}$ and if $N$ is a nodal set containing $C$, then there is a nodal set $N^{\prime}$ about $C$ contained in $N_{0}$ and disjoint from $h\left(\left[q_{0}, q\right]\right)$, where $q_{0}$ is the boundary point of $N$, which must then satisfy:

$$
N^{\prime} \cap W C h(N \cap W) \text {. }
$$

The function $h$ is continuous, as was shown in the proof of (i), so that $h\left(\left[q_{0}, q\right]\right)$ is compact and since it is contained in $W$ it is clear that we may choose a nodal set $N^{\prime}$ about $C$ disjoint from $h\left(\left[q_{0}, q\right]\right)$ and contained in $N_{0}$. From (i) we have that $N_{0} \cap W \subset h(W)$, and hence $N^{\prime} \cap W \subset N_{0} \cap$ $\cap W \subset h(W)=h(N \cap W) \cup h\left(\left[q_{0}, q\right]\right)$ from which it follows that $N^{\prime} \cap W$ $C h(N \cap W)$ since $\left(N^{\prime} \cap W\right) \cap h\left(\left[q_{0}, q\right]\right) \subset N^{\prime} \cap h\left(\left[q_{0}, q\right]\right)=\square$.

(iii) If $x_{1}, x_{2} \in C$ and $U_{1}$ and $U_{2}$ are open sets about $x_{1}$ and $x_{2}$, respectively, then there exists a nodal set $N$ of $S$ such that

$$
C \subset N \subset C^{(-1)} U_{1} \cap C^{(-1)} U_{2}
$$

Each of $C^{(-1)} U_{1}$ and ${ }^{*} C^{(-1)} U_{2}$ are open and $C C C^{(-1)} U_{1} \cap C^{(-1)} U_{2}$ because, in view of corollary $(2.4),\left\{x_{1}, x_{2}\right\} \subset C=C x$ for all $x \in C$. But
an open set about $C$ must contain a nodal set about $C$.

(iv) The mean is internal on $O$. Suppose that $x_{1} x_{2} \notin\left[x_{1}, x_{2}\right]$ for some $x_{1}, x_{2} \in C$ and assume that $\pi_{2} x_{1} \leqslant \pi_{2} x_{2}$, where $\pi_{2}$ is the second projection in the plane. As in the proof of proposition (3.1), let $V$ be a spherical neighborhood of $x_{1} x_{2}$ such that $V^{*} \cap\left\{x_{1}, x_{2}\right\}=\square$ and, using the continuity of the mean, let $U_{1}$ and $U_{2}$ be spherical neighborhoods of $x_{1}$ and $x_{2}$ respectively, such that $U_{1} U_{2} \subset V$ and $\left(U_{1} \cup U_{2}\right) \cap V=\square$. Using (iii), we let $N$ be a nodal set about $C$ such that $N C N_{0}$ and $N C C^{(-1)} U_{2} \cap$ $\frown C^{(-1)} V$ and then, using (ii), we let $N^{\prime}$ be a nodal set containing $C$ such that $N^{\prime} \cap W C h(N \cap W)$. Let $k$ be so large that $A_{k} C N^{\prime}$ and $A_{k} \cap U_{1}$ $\neq \square \neq A_{k} \cap U_{2}$ and choose $x_{1}^{\prime} \in A_{k} \cap U_{1}$ and $x_{2}^{\prime} \in A_{k} \cap U_{2}$.

From (i) we have that $x_{1}^{\prime}=r_{y_{1}}$ for some $y_{1} \in W, x_{2}^{\prime}=f_{y_{2}}$ for some $y_{2} \in W$ and because $x_{2}^{\prime} \epsilon A_{k} \subset N^{\prime} \cap W C h(N \cap W)$ we may suppose that $y_{2} \in N$ so that $C y_{2} \cap U_{2} \neq \square \neq C y_{2} \cap V$. It follows that $r_{y_{1}}=x_{1}^{\prime} \in C y_{2}$ $=\left[e_{y_{2}}, f_{y_{2}}\right]$ and hence, recalling that $f_{y_{2}}=x_{2}^{\prime} \in A_{k} \subset N_{0}$ we have that $C y_{2} \cap S y_{1}=\left[r_{y_{1}}, f_{y_{2}}\right]=\left[x_{1}^{\prime}, x_{2}^{\prime}\right]$ so that since, by lemma (3.2), each of $C y_{2} S y_{1}$ are submeans of $M$, so is $\left[x_{1}^{\prime}, x_{2}^{\prime}\right]$, from which we have that $x_{1}^{\prime} x_{2}^{\prime} \epsilon\left[x_{1}^{\prime}, x_{2}^{\prime}\right]$. But $x_{1}^{\prime} x_{2}^{\prime} \subset U_{1} U_{2} \subset V$ while $V \frown\left[x_{1}^{\prime}, x_{2}^{\prime}\right]=\square$, a contradiction.

Returning to the proof of the proposition, one obtains, from the internality of the mean on $C$, a contradiction as in the proof of proposition (3.1). Therefore our assumption that $W$ is an ideal of $S$ is false.

It may be remarked that in the preceding proof, the distributivity assumption was used only to get each of $C y$ and $S y$ a submean of $S$ for each $y \in W$ so we could have assumed that instead.

We next let $D=S \cup\{(r, s) \mid(2-r, s) \in S\}$ and again employ the notation $[A, B]$ for the continuum irreducible between $A$ and $B$, where $A$ and $B$ are subcontinua of $D$. Observe that $C_{1}=\{(r, s) \in D \mid r=0\}$, $C_{2}=\{(r, s) \in D \mid r=2\}$ and $W=\{(r, s) \in D \mid 0<r<2\}$ are the arccomponents of $D$.

(3.4) Propositron. $D$ admits no distributive mean.

Proof. Suppose, on the contrary that $D$ is a distributive mean. From the fact that each of $C_{1}, C_{2}$ and $W$ are the arc-components of $D$ we have that they are each submeans of $D$.

We establish a contradiction by the following series of assertions.

(i) Either $W C_{1} \subset C_{1}$ or $W C_{1} \subset W$ and, dually, either $W C_{2} \subset C_{2}$ or $W C_{2} \subset W$. Since $W C_{1}$ is arcwise connected and the arc-components of $D$ are $C_{1}, C_{2}$ and $W$, in order to show that $W C_{1} \subset C_{1}$ or $W C_{1} \subset W$, it suffices to show that $W C_{1} \not \subset C_{2}$. We know that $C_{1}^{[-1]}\left(D \backslash C_{2}\right)$ is open and contains $C_{1}$ because $C_{1}$ is compact and $C_{1}^{2} \subset C_{1}$ and hence $W \cap C_{1}^{[-1]}\left(D \backslash C_{2}\right) \neq \square$, i.e., $W C_{1}=C_{1} W \not \subset C_{2}$. The dual assertion follows similarly.

(ii) Either $W C_{1} \subset W$ or $W C_{2} \subset W$. If this were not true, then, in view of (i), we must have $W C_{1} \subset C_{1}$ and $W C_{2} \subset C_{2}$ and hence $D C_{1}=W^{*} C_{1}$ $C C_{1}$ and $D C_{2}=W^{*} C_{2} \subset C_{2}$ from which follows the impossible conclusion that both $C_{1} C_{2} \subset D C_{2} \subset C_{2}$ and $C_{1} C_{2}=C_{2} C_{1} \subset D C_{1} \subset C_{1}$. 
In view of the symmetry, we suppose for the remainder of the proof that $W C_{2} C W$ and consider the two possibilities $W C_{1} \subset C_{1}$ (and hence $\left.D C_{1} \subset C_{1}\right)$ and $W C_{1} \subset W$.

(iii) If $D C_{1} \subset C_{1}$, then $C_{2}=C_{2} x$ for all $x \in C_{2}$. If $x \in C_{2}$, then $D x$ is a subcontinuum of $D$ intersecting $C_{2}$, since $C_{2} x \subset C_{2}$, but not contained in $C_{2}$, since $W x \subset W C_{2} \subset W$ and hence, since $C_{2}$ is a $C$-set, we have that $C_{2} \subset D x$. It follows that $C_{2} \subset \mathrm{C}_{2} x$ (and hence $C_{2}=C_{2} x$ ) because, in addition to $W x \subset W$, we have that $C_{1} x \subset C_{1} D=D C_{1} \subset C_{1}$.

(iv) If $D C_{1} \subset C_{1}$, then, for each $y \in W, D y=\left[C_{1}, s_{y}\right]$ for some $s_{y} \in W$ and $W=\left\{s_{y} \mid y \in W\right\}$. It is clear that if $y \in W$, then $D y=\left[C_{1}, s_{y}\right]$ for some $s_{y} \in W$ and if we define $f: W \rightarrow W$ by $f(y)=s_{y}$, one shows that $f$ is continuous in a manner similar to that in the proof of assertion (i) of proposition (3.3) and hence it follows that $f(W)$ is connected.

If $y \in W$, then $D^{(-1)}\left\langle y, C_{2}\right]$ is open and contains $C_{2}\left(x \in C_{2} \Rightarrow x \in D x \cap\right.$ $\left.\cap C_{2} \subset D x \cap\left\langle y, C_{2}\right]\right)$ and, because $D$ is compact, $D^{[-1]}\left[C_{1}, y\right\rangle$ is open and contains $C_{1}\left(D C_{1} \subset C_{1} \subset\left[C_{1}, y\right\rangle\right)$, so if we let $y_{1} \in D^{[-1]}\left[C_{1}, y\right\rangle \cap W$ and $y_{2} \in D^{(-1)}\left\langle y, C_{2}\right] \cap W$, then $y \in\left[s_{y_{1}}, s_{y_{2}}\right] \subset f(W)$ since $f(W)$ is connected. We have therefore shown that $W \subset f(W)=\left\{s_{y} \mid y \in W\right\}$.

(v) If $W C_{1} \subset W$ and if $x_{1} \in C_{1}, x_{2} \in C_{2}$, then either $D x_{1} \cap C_{2}=\square$ or $D x_{2} \cap C_{1}=\square$. Since each of $C_{1}$ and $C_{2}$ are $C$-sets and since $D x_{1} \cap$ $\cap C_{1} \neq \square \neq D x_{2} \cap C_{2}\left(x_{1} \in D x_{1}, x_{2} \in D x_{2}\right)$, if we suppose that the assertion $s$ not true, we must have that $D x_{1}=D$ and $D x_{2}=D$. Each of $C_{1} x_{1}$, $W x_{1}$ and $C_{2} x_{1}$ are arcwise connected with $C_{1} x_{1} \subset C_{1}$ and $W x_{1} C W C_{1} \subset W$ so that since $D=C_{1} x_{1} \cup W x_{1} \cup C_{2} x_{1}$ and $C_{1}, C_{2}$ and $W$ are the arccomponents of $D$, it follows that $C_{2}=C_{2} x_{1}$. We then have $x_{1} x_{2} \in C_{2}$ and a dual argument shows that $x_{1} x_{2} \in C_{1}$ which is impossible.

If $D C_{1} C C_{1}$, by utilizing (iv) and our assumption that $W C_{2} \subset W$ one can show as in the proof of proposition (3.3) that the mean is internal on $C_{2}$ which, again as in the proof of proposition (3.3), will then contradiet (iii).

If $W C_{1} \subset W$ (and $W C_{2} \subset W$ ), then, in view of $(v)$ and the symmetry we may suppose that $D x_{1} \cap C_{2}=\square$ with $x_{1} \in C_{1}$. Then $D x_{1}$ is a continum contained in $C_{1} \cup W$ which intersects both $C_{1}$ and $W$ so that since $C_{1}$ is a $C$-set, it contains $C_{1}$ and hence is homemorphic to the sinusoidal continuum $S$ defined earlier. Furthermore, $D x_{1}$ is a submean of $D$ such that $\left(D x_{1} \cap W\right) C_{1} \subset D x_{1} \cap W$ so that if we apply proposition (3.3) we are led to a contradiction.

Having shown that all possibilities lead to a contradiction, the proposition follows.

4. We have just seen that $D$ admits no distributive (and hence no medial) mean. However, each of the following questions awaits an answer.

(4.1) Does $D$ admit a mean?
(4.2) Does S admit a mean? a distributive mean? a medial mean?

It is easy to show (see Aumann [2]) that any retract of a space admitting a mean must admit a mean. Hence, if $S$ admits no mean, neither does $D$. For results relevant to the medial case of $(4.2)$, the reader is referred to Sigmon [7].

\section{References}

[1] J. Aczél, Lectures on Functional Equations and their Applications, New York 1966.

[2] G. Aumann, Über Räume mit Mittelbildungen, Math. Ann. 119, (1943); pp. $210-215$.

[3] J. M. Day and A. D. Wallace, Semigroups acting on continua, J. Austral. Math. Soc., to appear.

[4] B. Eckmann, Räume mit Mittelbildungen, Comment. Math. Helv. 28 (1954), pp. 329-340.

[5] B. Eckmann, T. Ganea and P. J. Hilton, Generalized means, from Studies in Mathematical Analysis and Related Topics, Stanford 1962.

[6] Orrin Frink, Symmetric and self-distributive systems, Amer. Math. Monthly 62 (1955), pp. 697-707.

[7] Kermit Sigmon, Medial topological groupoids, Aequationes Mathematicae, to appear.

[8] A. D. Wallace, The position of $C$-sets in semigroups, Proc. Amer. Math. Soc. 6 (1955), pp. 639-642.

UNIVERSITY OF FLORIDA

Gainesville, Florida

Regu par la Rédaction le 5. 7. 1967 